\title{
The limits of prior entry: Nonsensitivity of temporal order judgments to selective preparation affecting choice reaction time
}

\author{
CLAUDE VANDERHAEGHEN and PAUL BERTELSON \\ Laboratoire de Psychologie expérimentale, Université libre de Bruxelles \\ 117, av. Ad. Buyl, 1050 Bruxelles, Belgium
}

\begin{abstract}
On each trial, a reference tactile stimulus and another stimulus, which was unpredictably either a sound or a light-pulse, were presented with a variable time interval. The subject had to give a fast choice reaction to the uncertain stimulus and also to judge its temporal position relative to the reference stimulus. Manipulation of the relative frequency of occurrence of the uncertain stimuli affected choice reaction time in the usual way, but produced unsystematic effects on apparent order. It is concluded that selective preparation which influences response latency does not necessarily produce a prior entry effect.
\end{abstract}

The principle of prior entry is one of the best remembered generalizations of early experimental psychology. It held that the perceived order of occurrence of closely spaced events can be affected by preliminary adjustments of attentional mechanisms (Titchener, 1908; Wundt, 1911). The principle is still often quoted and generally believed to have been based on well established facts (e.g., Reber \& Anderson, 1970; Kahneman, 1973, p. 137). Yet, examination of the literature shows a surprising paucity of supporting data. Until the recent paper by Sternberg, Knoll and Gates (1971), to be discussed below, the evidence in favor of the notion consisted exclusively of the effects of instructing observers to attend selectively to one of two sources of stimulation: Titchener (1908) contented that estimiation of the position occupied by the arm of a metronome at the time the stroke occurred could be influenced in that way, and Stone (1926) reported similarly produced shifts of the point of subjective simultaneity of two discrete stimuli. This procedure, where the observers were requested to adopt a particular mental set, without any objective criterion of success, is of course of dubious significance.

The possibility of biasing processing time in favor of one of a set of alternatives through prior adjustments has, on the other hand, been extensively documented in the choice reaction situation. In that situation, selective preparation for one alternative can be induced either by increasing its relative frequency of occurrence (Fitts, Peterson, \& Wolpe, 1963) or by increasing the payoff associated with fast reactions (LaBerge, Tweedy, \& Ricker, 1967). We have undertaken a set of experiments

*The present work has been subsidized by the Belgian Fonds de la Recherche Fondamentale Collective under Contract 10.152 The first author held an "Aspirant" grant from the Belgian Fonds National de la Recherche Scientifique. Thanks are due to Saul Sternberg, Ronald Knoll and John Mollon for useful comments on a previous version of this paper. to examine if these manipulations can also affect temporal order judgments (TOJ). An answer to that question could help in identifying that or those components of processing time which are susceptible to selective preparation effects. The rationale of this kind of approach has recently been analyzed in a very thorough way by Sternberg and Knoll (1973).

In a first attempt at producing a prior entry effect, Bertelson and Tisseyre (1969) had subjects judge the order of occurrence of a click and a tachistoscopically presented word, and found that the judgments were not influenced by the relative frequency of presentation of the word. They concluded that TOJ was based on evidence gathered before the stage affected by word frequency, which they assumed to be a stimulus identification stage. Considering that the existence of a common preidentification stage was unlikely with stimuli pertaining to different modalities, other attempts were made where the uncertainty concerned the modality reached by the stimuli submitted to TOJ. In an unpublished pilot experiment, the first author collected TOJs for pairs of stimuli, one of which was always in the tactile modality while the other was unpredictably either visual or auditory. Manipulations of the selective frequency of occurrence of the uncertain stimulus had nonconsistent effects on the judgments.

Such negative results, however, do not necessarily demonstrate a lack of sensitivity of apparent order to selective preparation. It can also be argued that the subjects did not consistently prepare for the preponderant event, because the TOJ task provides no incentive for doing so. In a reaction task, the subject is rewarded for preparing for the most frequent alternative by the achievement of faster reactions, while order judgments have no perceptible consequences for him. One way out of that difficulty consists of requiring both choice reactions and TOJs on the same trials. The 
reaction task might then provide both an incentive to prepare consistently and a way of assessing preparation. The experiment to be reported used the same stimulating situation as the just mentioned pilot experiment. Two stimuli were presented on each trial, one of which, the reference stimulus, was always tactile while the other was unpredictably a light or a sound pulse. The subject had to give a TOJ and to identify the uncertain stimulus by pressing one of two keys. Different frequencies of occurrence of the uncertain stimuli were used. If the subject changed his preparation strategy accordingly, the RTs would be affected. It could then be established unambiguously if these adjustments affect TOJ also.

The principle of both inducing and indexing preparation by associating a reaction task with TOJ has recently been applied by Sternberg, Knoll, and Gates (1971) in an experiment where, on experimental trials, subjects judged the order of occurrence of two stimuli (a sound and a tap) and reacted to one of them. These trials were mixed with catch trials, where the reaction stimulus was omitted, so that the reaction task was of Donder's c-type. The point of subjective simultaneity was affected by the reaction instructions in five of the six subjects. The result is, for the time being, the strongest available demonstration of a prior entry effect. On the other hand, it does by no means imply that a similar effect should be obtained in the present study, since, as the authors remark (Note 11 ), the preparatory adjustments induced by the c-reaction task may be different from those caused by frequency unbalance in b-reactions.

\section{METHOD}

\begin{abstract}
Apparatus
All sources of experimental stimuli were concentrated in a single console-shaped chassis situated on a table, in front of the subject, in his median plane. The tactile stimulation occupied the low anterior part of the console, which was $2 \mathrm{~cm}$ high, with a sagitally oriented slot shaped to accommodate the right index finger of the subject. The stimulation was provided by the arm of a Stoelting SA-16 galvanometric recording pen, which, when activated, moved horizontally and came to hit gently the left side of the finger. The activating signal was a $10-\mathrm{msec}, 8-\mathrm{V}$ impulse. The auditory stimulator was a small $3 \mathrm{w} / 800 \mathrm{ohm}$ loudspeaker, hidden inside the chassis, which could be heard through an opening in the vertical anterior wall, about $5 \mathrm{~cm}$ above the tactile stimulator. The auditory stimuli were produced by feeding it with a $1-\mathrm{msec}, 5-\mathrm{V}$ impulse. The visual stimulator was a light emitting diode (Monsanto Type MV 50) situated between the tactile stimulator and the opening. The visual stimuli were produced by feeding it with the same $1-\mathrm{msec}, 5-\mathrm{V}$ impulse. A neon pea lamp, situated on top of the chassis, immediately above the opening, served to deliver warning signals. All signals were produced by Tektronix 161 pulse generators, which were controlled by a Tektronix 162 waveform generator. The reaction keys were placed towards the left of the stimulating chassis. The subject activated them with the index and middle fingers of the left hand. RTs were measured on a TC12 advance electronics timer-counter.
\end{abstract}

\section{Subjects}

Five students volunteered to participate in six sessions. They were paid a fixed hourly rate plus a bonus depending on performance in the reaction task.

\section{Procedure}

On each trial, two stimuli were presented. One was the tap on the finger, which served as reference stimulus. The other, the uncertain stimulus, was unpredictably either the light or the sound pulse. The two stimuli were separated by a time interval which varied over the range -120 to $+120 \mathrm{msec}$. The beginning of each trial was signaled by a $20-\mathrm{msec}$ pulse in the warning neon lamp, always $1 \mathrm{sec}$ before the occurrence of the tactile stimulus.

The subject had two tasks to perform: (1) choice reaction: responding to the onset of the uncertain stimulus by pressing one of the two response keys, one for the visual, the other for the auditory stimulus; interspersed randomly among the experimental trials were $18 \%$ of catch-trials where only the tactile stimulus occurred, and the correct response was abstention; (2) Temporal order judgments: naming the stimulus of the presented pair which had been presented first.

Three conditions were studied, which differed by the relative frequencies of occurrence of the uncertain stimuli on experimental trials: Condition A75: $25 \%$ of visual, $75 \%$ of auditory stimuli; Condition A50: equal proportions of both; Condition A25: $75 \%$ of visual, $25 \%$ of auditory stimuli. At each session, 66 trials were given under each of the three conditions. Only the results of the last four sessions were analysed.

After each correct response, the subject was told whether it had been fast or slow, meaning faster or slower than the mean reaction time of the previous session. After an error, no information regarding speed was communicated. The bonus paid to the subject was based on the number of fast correct reactions and the number of errors ( 1 error $=4$ fast reactions).

\section{RESULTS AND DISCUSSION}

Individual points of subjective simultaneity (PSS), i.e., the time interval giving 50\% of "touch before" responses, were calculated separately for each uncertain stimulus and every condition, by linear interpolation on psychometric functions after transformation of the proportions into $\mathrm{z}$-scores.

Mean correct RTs which appear in the upper part of Figure 1 show the usual relation to stimulus relative frequency in four of the five subjects: RTs to the auditory stimulus decrease from Condition A25 to Condition A75, while those to the visual stimulus increase from A25 to A75. Subject 5's reaction speed is not affected systematically by stimulus frequency. This finding must not be considered as too disturbing: in experiments dealing with the effects of frequency bias, there is often a minority of subjects who do not take advantage of the opportunity to prepare selectively. On the other hand, our main question, whether prior adjustments to frequency unbalance which affect RT also affect TOJ, can only be tested on the responses of subjects 14 whose RTs are affected.

As appears in the lower part of Figure 1, in each of these four subjects the PSSs for both visual and auditory stimuli tend to shift to later values with increasing proportions of auditory stimuli. In other words, the pattern predicted on the basis of prior entry is observed for auditory stimuli, and the opposite pattern for visual stimuli. Whatever the reason for this puzzling picture, there is no way to relate it to the effect observed on RT. 


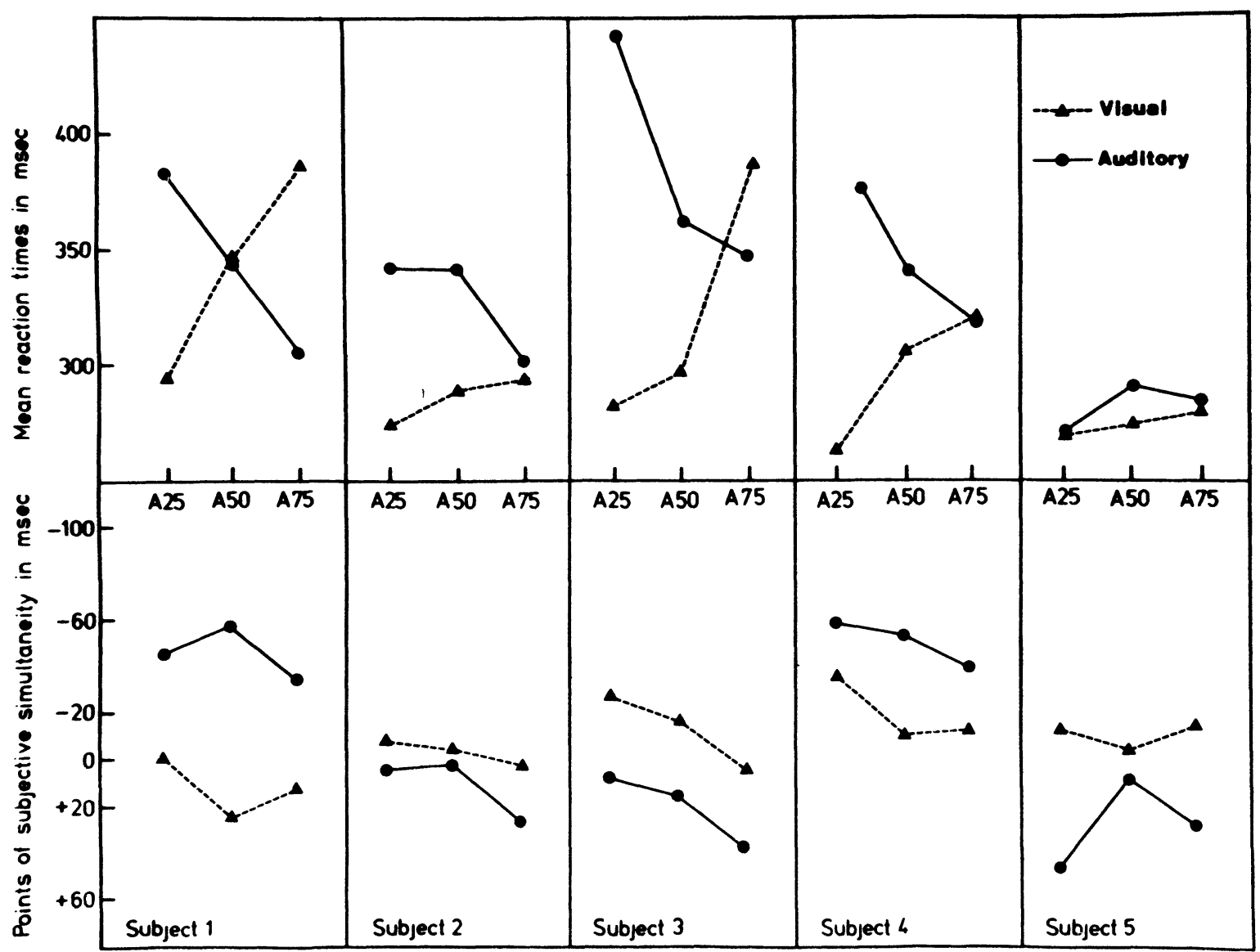

Figure 1. Mean correct reaction times (above) and points of subjective simultaneity (below) as functions of conditions, for each uncertain stimulus. A positive PSS means that the uncertain stimulus must come later than the reference tactile stimulus to be judged as simultaneous. The ordinate for the PSS has been been drawn with the negative end above, so that support for the prior entry prediction would be indicated by RT and PSS lines running parallel to each other.

The main conclusion is, thus, that selective preparation to give a fast identification reaction to one particular stimulus does not necessarily affect the apparent time of occurrence of that stimulus. Previous failures to produce a prior entry effect through frequency unbalance manipulations were not due to lack of incentive to prepare selectively.

As in other cases of failures of cross predictions between RT and TOJ (e.g., Gibbon \& Rutschmann, 1969; Sanford, 1971) the result can be interpreted in several ways.

One possibility is that the processing stage of RT which is affected by frequency unbalance is being bypassed in TOJ. Several suggestions have been made regarding the function of that stage. Starting from the now well established fact that, in choice tasks with a many-to-one mapping, preparation effects are largely stimulus-specific, even if some degree of generalization to other stimuli mapped onto the same response has sometimes been found (e.g., LaBerge, Legrand, \& Hobbie, 1969), several authors have argued that selective preparation influences a stimulus identification stage (e.g., Bertelson \& Tisseyre, 1966; Hawkins, Thomas, \&
Drury, 1970; Hinrichs \& Krainz, 1970). Adopting that viewpoint, Bertelson and Tisseyre (1969) concluded that TOJ was not affected by stimulus frequency because it is based on the outputs of a preidentification detection stage. The reasoning cannot be extended to the present situation, where it would imply the implausible notion of a stage where information is available that either a flash or a click has occurred, but it cannot be stated which it was. In the framework of the identification stage interpretations of selective preparation, the present results would lead to the notion of parallel independent analyzing stages, respectively for RT and TOJ.

An alternative suggestion regarding the locus of selective preparation, which would accommodate the present results beautifully has been made recently by Keele $^{1}$ and by Hawkins, McKay, Holley, Friedin, and Cohen (1973). The relevant stage would be one concerned with response selection, where information favoring each response is being accumulated separately for each stimulus. ${ }^{2}$ The main problem for that interpretation is that it predicts an interaction with $S-R$ compatibility which Sanders (1970) first reported, but which later work by Hawkins and his collaborators 
(reviewed in Hawkins et al., 1973) showed to be a rather elusive phenomenon.

There is also a completely different possible interpretation, which dispenses with the notion of processing stages. TOJ and RT can be based on different features of the same internal representations of the stimuli, and only the feature critical for RT be affected by preparation. This suggestion is explicated in detail by Sternberg and Knoll (1973, pp. 648-650).

The confrontation of our result with that of Sternberg, Knoll, and Gates (1971) implies that the adjustments induced by frequency unbalance, which do not affect apparent order, are different from those involved in their c-reaction task, which do affect it. Further work is needed to analyze the difference. It seems particularly desirable to try tn produce both adjustments within the same experimental situation.

\section{REFERENCES}

Bertelson, P., \& Tisseyre, F. Choice reaction time as a function of stimulus versus response relative frequency of occurrence. Nature, 1966, 212, 1069-1070.

Bertelson, P. \& Tisseyre, F. Apparent order and stimulus uncertainty. Psych onomic Science, 1969, 15, 65-66.

Fitts, P. M., Peterson, J. R., \& Wolpe, G. Cognitive aspects of information processing: II. Adjustments to stimulus redundancy. Journal of Experimental Psychology, 1963, 65, 423-432.

Gibbon, J., \& Rutschmann, R. Temporal order judgments and reaction time. Science, $1969,165,413-415$.

Hawkins, H. L., McKay, S. L., Holley, S. L., Friedin, B. D., \& Cohen, S. L. Locus of the relative frequency effect in choice reaction time. Journal of Experimental Psychology, 1973, $101,90-99$

Hawkins, H. L., Thomas, G. B., \& Drury, K. B. Perceptual versus response bias in discrete reaction time. Journal of Experimental Psychology, 1970, 84, 514-517.
Hinrichs. J. V.. \& Krainz, P. L. Expectancy in choice reaction time: Stimulus or response anticipation? Journal of Experimental Psychology, 1970, 85, 330-334.

Kahneman, D. Attention and effort. Englewood Cliffs: Prentice-Hall, 1973.

LaBerge, D., Legrand, R., \& Hobbie, R. K. Functional identification of perceptual and response biases in choice reaction time. Journal of Experimental Psychology, 1969, 79, 295-299.

LaBerge, D., Tweedy, J., \& Ricker, J. Selective attention: Incentive variables and choice time. Psychonomic Science, 1967, 8, 341-342.

Reber, A. S., \& Anderson, J. R. The perception of clicks in linguistic and nonlinguistic messages. Perception and Psychophysics, 1970, 8, 81-89.

Sanders, A. F. Some variables affecting the relation between relative stimulus frequency and choice reaction time. Acta Psychologica, 1970, 33, 45-55.

Sanford, A. J. Effects of changes in the intensity of white noise on simultaneity judgments and simple reaction time. The Quarterly Journal of Experimental Psychology, 1970, 23, 296-303.

Sternberg, S, \& Knoll, R. L. The perception of temporal order: fundamental issues and a general model. In: Kormblum, $S$. (Ed), Attention and performance IV, 1973, New York: Academic Press, 629-685.

Sternberg, S., Knoll, R. L. \& Gates, B. A. Prior entry reexamined: effects of attentional bias on order perception. Paper presented at Annual Meeting of Psychonomic Society, St. Louis, 1971.

Stone, S. A. Prior entry in the auditory-tactual complication. The American Journal of Psychology, 1926, 37, 284-287.

Titchener, E. B. Lectures on the elementary psychology of feeling and attention. New York: MacMillan, 1908.

Wundt, W. Grundzuge der physiologischen Psychologie, Vol. 3. Leipzig: Engelmann, 1911.

\section{NOTES}

1. Keele, $S$ Personal communication, 1973.

2. Generalization to all stimuli calling for the same response demands an additional explanatory principle under both hypotheses, and is thus not critical for discriminating between them, contrary to what Hawkins et al suggest.

(Received for publication September 17, 1974.) 